\title{
Photoinduced blinking in a solid-state quantum system
}

\author{
Amanuel M. Berhane, Carlo Bradac, and Igor Aharonovich* \\ School of Mathematical and Physical Sciences, University of Technology Sydney, Ultimo, New South Wales 2007, Australia
}

(Received 19 March 2017; published 12 July 2017)

\begin{abstract}
Solid-state single-photon emitters (SPEs) are one of the prime components of many quantum nanophotonics devices. In this work, we report on an unusual, photoinduced blinking phenomenon of SPEs in gallium nitride. This is shown to be due to the modification in the transition kinetics of the emitter, via the introduction of additional laser-activated states. We investigate and characterize the blinking effect on the brightness of the source and the statistics of the emitted photons. Combining second-order correlation and fluorescence trajectory measurements, we determine the photodynamics of the trap states and characterize power-dependent decay rates and characteristic "off"-time blinking. Our work sheds light into understanding solid-state quantum system dynamics and, specifically, power-induced blinking phenomena in SPEs.
\end{abstract}

DOI: 10.1103/PhysRevB.96.041203

Fluorescence blinking, also referred to as fluorescence intermittency, is usually an undesired but ubiquitous phenomenon in most quantum light sources, including quantum dots [1-3], defects in wide-band-gap semiconductors [4-6], and single molecules [7-9]. Blinking arises when, upon laser excitation, a fluorescent center undergoes sporadic jumps between "dark" and "bright" states in the photoemission [3]. This phenomenon is identified by the random fall ("off"/"dark" state) and rise ("on"/"bright" state) in photon counts during long-time (milliseconds to hours) fluorescence photostability measurements. Although the cause of the "dark" state has been rigorously studied in various fluorescent systems, a universal physical mechanism that explains blinking has not been pinned down yet [2,10-14].

Recently, a new family of single-photon emitters (SPEs) in gallium nitride $(\mathrm{GaN})$ has been discovered [15]. Using both experimental and modeling techniques, the single-photon emission was attributed to the recombination of localized excitons to a point defect sitting near or inside a cubic inclusion. These emitters show bright, narrow-band emission with linear polarization, which is suitable for quantum information applications. Under continuous-wave laser excitation, the vast majority of the emitters display photostable fluorescent emission with single state photon statistics. Interestingly however, approximately $5 \%$ of the emitters start showing blinking once the power of the excitation laser rises over a certain threshold.

In this work, we investigate the nature of this excitationinduced blinking behavior of SPEs in GaN at room temperature. Unlike most known SPEs where blinking occurs across all excitation powers without altering its photodynamics, in the present work we report on a previously unexplored behavior where blinking only occurs above a particular excitation threshold and the emitter's photodynamics is permanently altered without bleaching. By combining transition kinetics analysis and fluorescence correlation measurements at short (nanoseconds) and long (millisecond) time scales, we gather insights into the blinking mechanism. Furthermore, we propose a mechanism to explain this behavior in an attempt to

*igor.aharonovich@uts.edu.au generalize the phenomenon and extend the description of such laser-induced blinking to other solid-state SPEs.

The sample used in this study is a $2-\mu \mathrm{m}$-thick magnesiumdoped GaN layer on 2- $\mu \mathrm{m}$ undoped GaN grown on sapphire. The SPEs were isolated at room temperature (RT) using a custom-made confocal microscope [15] equipped with a Hanbury Brown and Twiss (HBT) interferometer for secondorder autocorrelation $\left[g^{2}(\tau)\right]$ measurements. A 532-nm, cw laser was used for excitation focused to a spot size of $\sim 450 \mathrm{~nm}$ using a 0.9 numerical aperture objective and the laser power was measured at the entrance pupil of the objective (filling factor $\sim 2$ ).

Figure 1(a) shows the photoluminescence spectrum of an isolated SPE excited with $200 \mu \mathrm{W}$ of laser power, at room temperature. The emitter displays a characteristic emission with zero-phonon line (ZPL) at $647 \mathrm{~nm}$ and full width at half maximum (FWHM) of $\sim 4 \mathrm{~nm}$. Figure 1(b) shows the emitter's fluorescence stability, measured at $3 \mathrm{~mW}$. The corresponding occurrence statistics of the emission intensity is shown in Fig. 1(c). The photon distribution follows a single state photon statistics at excitation power of $3 \mathrm{~mW}$ [16].

For this particular center, a 5-mW excitation induced a sudden change in the photon statistics, which starts displaying a marked blinking behavior as illustrated in Fig. 1(d). The photon statistics of the emitter at $5 \mathrm{~mW}$ [Figs. 1(d) and 1(e)] shows three distinct states, in contrast to the single state photon statistics of the same emitter at $3 \mathrm{~mW}$ [Figs. 1(b) and 1(c)]. We note that this blinking did not result in bleaching of the emitter during the time frame of this characterization. The fluorescence trajectories-before and after the blinking is induced-for different excitation intensities are shown in Fig. S2 [17]. The average intensity values of the emitter before and after blinking are given in Table $\mathrm{S} 1$.

Prompted by the unusual blinking characteristics of the SPEs, the $g^{2}(\tau)$ measurements were taken at different excitation powers before and after blinking, using an HBT interferometer. Figure 1(f) shows two $g^{2}(\tau)$ curves of the same SPE taken at excitation power of $100 \mu \mathrm{W}$ before (red) and after (blue) blinking was induced. The autocorrelation curves in Fig. 1(f) are replotted in two separate panels and are presented in Figs. S3(b) and S3(c) for better visibility of the change in the absolute antibunching characteristics (see Supplemental Material [17]). In addition, a sequence diagram 


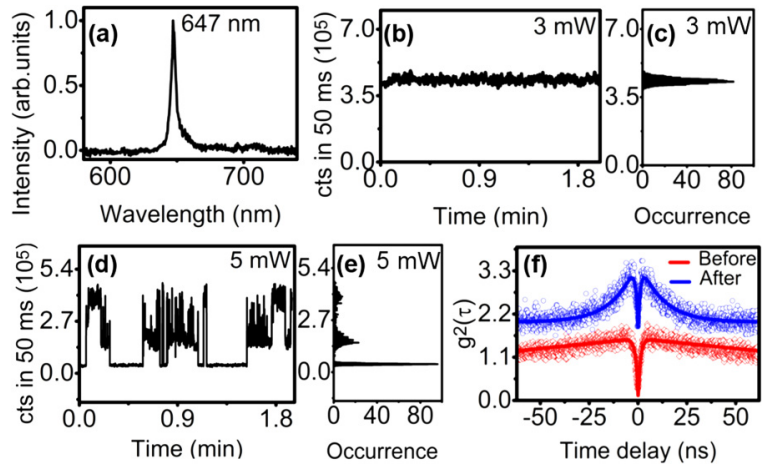

FIG. 1. Excitation power-induced blinking of a SPE in GaN. (a) RT spectra of the SPE taken under $200-\mu \mathrm{W}$ power excitation; the ZPL lies at $647 \mathrm{~nm}$ with a FWHM of $\sim 4 \mathrm{~nm}$. (b) Fluorescence trajectory of the same emitter. The time trace is collected from the ZPL with $630 \pm 30 \mathrm{~nm}$ bandpass filter for $2 \mathrm{~min}$. (c) Occurrence statistics of the number of photon counts in (b) over a time of $2 \mathrm{~min}$. The emitter shows stable emission. (d) Fluorescence trajectory of the same emitter excited with $5 \mathrm{~mW}$. The time trace is collected from the ZPL using the same bandpass filter. (e) Photon occurrence statistics of (d) at the same excitation power of $5 \mathrm{~mW}$, with notable blinking. Time binning in (a)-(e) is $50 \mathrm{~ms}$. (f) The $g^{2}(\tau)$ measured for the same emitter before (red) and after (blue) the blinking was induced with high-power excitation; the $g^{2}(\tau)$ curves in (f) are taken with $100 \mu \mathrm{W}$ excitation power. The blue curve is offset vertically by 1 for clarity (see Fig. S3 of the Supplemental Material [17]). The same emitter yields two different values of $g^{2}(0) \approx 0.24$ vs $g^{2}(0) \approx 0.65$ for before and after the high-power-excitation blinking was induced, respectively.

is provided in Fig. S1 [17] to illustrate how the measurement was carried out. Remarkably, the photon statistics before and after the induced blinking are different, with the emitter showing - beyond the expected bunching at intermediate time scales due to the high excitation power-a reduction in the contrast of the $g^{2}(0)$ function $\left[g^{2}(0)=0.65\right.$ after, vs 0.24 before]. This indicates that the transition dynamics of the emitter is permanently modified. We argue that the change is caused by the activation of a trap state which provides an additional, nonradiative transition pathway to the ground state, before the system can be reexcited (level diagram is shown in Fig. S3 of the Supplemental Material [17] for illustration) $[18,19]$. Note that this behavior is dramatically different from that of other solid-state emitters-e.g., the nitrogen-vacancy center in diamond-where high excitation simply results in an increased population of its metastable state, and the photodynamics is preserved [20-22].

While the presence of a newly photon-activated state is revealed via reduced average intensity and $g^{2}(0)$ value after blinking, the three states blinking observed in Figs. 1(d) and 1 (e) can no longer be resolved via the 50-ms fluorescence trajectory measurement [17]. This is due to fast decay from state 4 to the ground state via strongly coupled decay coefficient $\kappa_{41}$ making the "off"-state duration much shorter to be detected by a $50-\mathrm{ms}$ bin. One mechanism that facilitates fast depopulation from a trap state is the auger-assisted decay process, where the subsequent addition of a charge state knocks the former to the ground state [23-25]. This is consolidated (a)

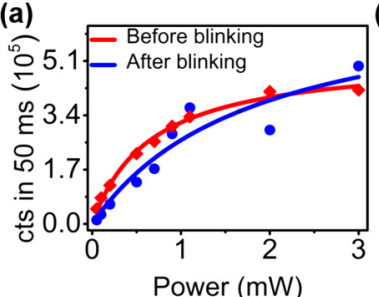

(b)
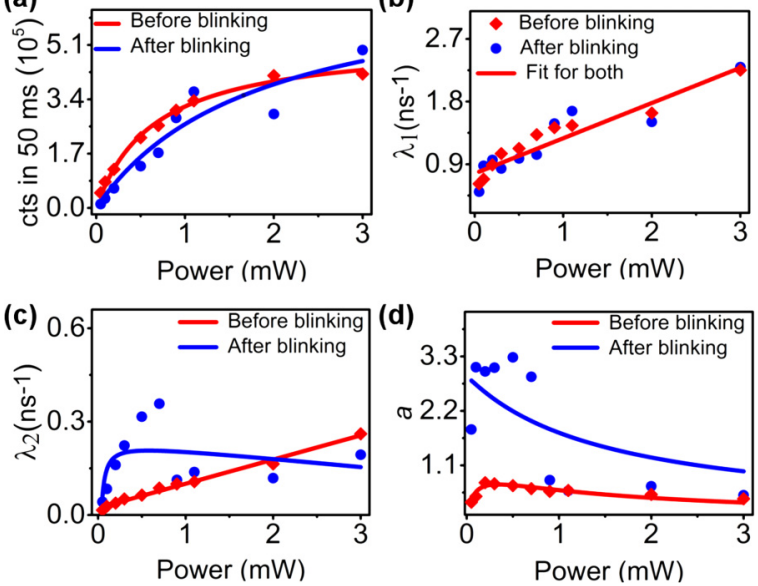

FIG. 2. Excitation-power-dependent parameters of the emitter. (a) Brightness of the emitter before (red) and after (blue) blinking is shown as the average photon counts at different powers. Before blinking, at saturation power $\left(P_{\text {sat }}\right) \sim 660 \mu \mathrm{W}$, the highest intensity of $527 \mathrm{kcounts} / \mathrm{s}$ is obtained. After blinking, the saturation behavior is fitted with a three-level model showing a remarkably different curve. (b)-(d) Power-dependent characteristics for the fit parameters $\tau_{1}, \tau_{2}$, and $a$, respectively, for the $g^{2}(\tau)$ function. These values are extracted as parameters from the $g^{2}(\tau)$ function fitting (Supplemental Material, Fig. S5 [17]). A three-level model with linear power dependence for the shelving state accurately described the transition kinetics before blinking (red fitting lines). After blinking, however, the same model fails to fit $\lambda_{2}$ and $a$ as highlighted by the blue lines in (c) and (d).

by the three distinct photon states shown Figs. 1(d) and 1(e) where multilevel blinking is due to the power-law distribution as a result of a fluctuating nonradiative process.

The background-corrected $g^{2}(\tau)$ curves in Fig. 1(f) are fitted with a three-level model given in Eq. (1).

$$
g^{2}(\tau)=1-(1+a) e^{-\left[\lambda_{1} \tau\right]}+a e^{-\left[\lambda_{2} \tau\right]},
$$

where $\lambda_{1}$ and $\lambda_{2}$ are fitting parameters for radiative and nonradiative decay rates while $a$ is a scaling factor for bunching. With an excitation power of $100 \mu \mathrm{W}, g^{2}(0)=0.24$ and 0.65 "before" and "after" blinking, respectively. The deviation from zero is due to background fluorescence which is relatively strong in $\mathrm{GaN}$ from other impurities. While the contrast in $g^{2}(0)$ is reduced after the power-induced blinking has occurred, we believe it is still associated with the same single emitter, only with a much lower signal-to-noise ratio (i.e., reduced brightness as per the additional dark shelving state). Additional power-dependent $g^{2}(\tau)$ curves before and after blinking are shown in Fig. S5 (see Supplemental Material [17]).

To further study the changes in transition kinetics of the emitter, we conduct an analysis of brightness and rate the coefficient before and after blinking. Figure 2(a) shows plots of power-dependent intensity values for the SPE before and after the blinking was induced. The plots are fitted with a three-level system model, and the intensity is given by $I=I_{\infty} P /\left(P+P_{\text {sat }}\right)$ where $P_{\text {sat }}$ is the saturation power and $I_{\infty}$ is the highest intensity obtained. Before blinking, $I_{\infty}$ is $\sim 527 \mathrm{kcounts} / \mathrm{s}$ at $P_{\text {sat }} \sim 660 \mu \mathrm{W}$. The same center showed two different saturation behaviors before and after blinking. After the blinking is induced, the emission intensity 
at excitation powers $<900 \mu \mathrm{W}$ is slightly lower than it was before blinking (for the same powers). This is consistent with the model we propose of a laser-activated trap state compounding the nonradiative transition. At excitation powers $\geqslant 900 \mu \mathrm{W}$, the effect of the additional trap state on emission intensity is overall reduced due to rapid depopulation to the ground state [26,27].

The transition kinetics analysis is carried out by extracting $\lambda_{1}, \lambda_{2}$, and $a$ as fit parameters from the background-corrected, power-dependent $g^{2}(\tau)$ measurements before and after blinking as shown in Figs. S4(a) and S4(b). Figures 2(b) and 2(d) display the extracted $\lambda_{1}, \lambda_{2}$, and $a$ as a function of excitation powers, before and after blinking was induced. The power dependence of $\lambda_{1}, \lambda_{2}$, and $a$ is fitted by assuming a three-level model with a shelving state that depends linearly on the excitation power for both "before" and "after" blinking (see Supplemental Material [17] for detailed analysis) [28-30]. The parameter $\lambda_{1}$ before and after blinking remains unchanged, hinting that the radiative decay pathway is unaffected by the blinking. Note that the ZPL in the spectra remains unchanged after blinking was induced as shown in Fig. S3(a) [17], suggesting that whatever the nature of the laser-induced change in the emitter might be, such change does not alter, in a detectable manner, the first excited electronic state. Conversely, there is a clear difference in the distribution of values for $\lambda_{2}$ and $a$, where the three-level model for the emitter fails to fit the power-dependent behaviors of $\lambda_{2}$ and $a$ after blinking. This is also in accord with the pronounced bunching behavior at intermediate time scales observed for the emitter at low powers after the induced blinking.

While rate analysis and brightness characterization hint to a permanent change in the photodynamics of the emitter, a more direct evidence for the power-induced trap state is required. We therefore recorded long-time fluorescence correlation behaviors for two different emitters in the time range of a few microseconds to $0.1 \mathrm{~s}$ - one that exhibits absolute photostability and another one that exhibits blinking at higher excitation powers (similar to the one characterized earlier). The spectra and saturation behaviors of the two SPEs is shown in Fig. S6 (see Supplemental Material [17]). Figure 3(a) shows power-dependent, long-time-scale correlated $g^{2}(\tau)$ from the photostable SPE. Each measurement is fitted with an exponential decay function that holds the least $\chi^{2}$ value, where the decay rate determines the bunching behavior [31]. In this case, no significant (compared to noise level) decay rates can be observed at the microsecond-to-millisecond time scale and the $g^{2}(\tau)$ is fitted with a single exponential of the form $g^{2}(\tau)=1+A e^{-\left[\lambda_{2} \tau\right]}$ to extract the decay rate, $\lambda_{2}$, from the shelving state in the nanosecond range at different powers. Note that the radiative decay rate, $\lambda_{1}$, is not shown in this measurement as correlation starts from a few microseconds. As shown, the $g^{2}(\tau)$ remains constant along the normal line during the measurement time for all excitation powers. Thus, the stable emitter is fully described by a three-level model showing no additional trap state at long correlation time.

The same set of measurement and analysis is carried out for an emitter that exhibited excitation-dependent blinking. In this case, the $g^{2}(\tau)$ measurement at long time scales show a strong dependence on the excitation power as evident from Fig. 3(b), where an extra decay channel appears at the
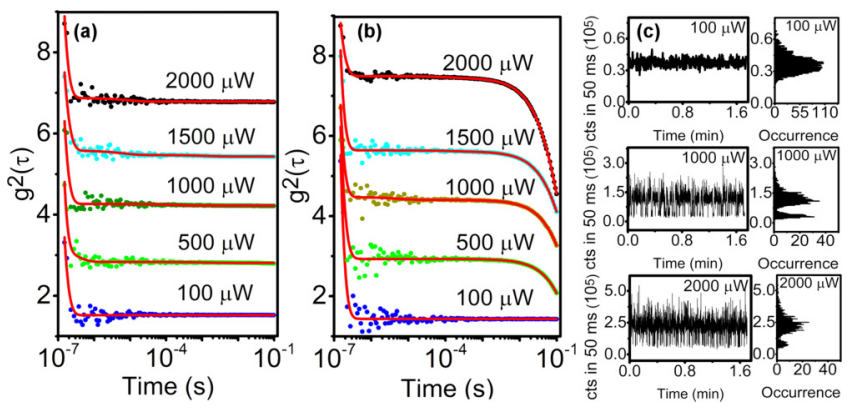

FIG. 3. Power-dependent long-time $g^{2}(\tau)$ characteristics. (a) Long-time-scale, power-dependent $g^{2}(\tau)$ characteristics of a stable emitter. The best fit is determined using a single exponential decay function with the least $\chi^{2}$ value. $g^{2}(\tau)$ starts with monotonic decay that corresponds to nanosecond shelving state but remains constant for the measurement time scale range of microseconds to 0.1 s. (b) Similar long-time-scale $g^{2}(\tau)$ functions of the blinking emitter at different excitation powers. Fitting the $g^{2}(\tau)$ characteristics at excitation powers of $100 \mu \mathrm{W}$ is done using a single exponential decay function where $g^{2}(\tau)$ remained constant along the normal; whereas, for excitation powers of $500-2000 \mu \mathrm{W}$, the best fit is a double exponential decay function with the $g^{2}(\tau)$ exhibiting an additional decay in the millisecond range. Individual plots in (a) and (b) are shifted for clarity. (c) Fluorescence trajectory and photon occurrence statistics under 50-ms binning for the emitter in (b) with increasing excitation power. The initially stable emitter starts blinking for excitation powers $\geqslant 500 \mu \mathrm{W}$ showing two distinct intensity traces.

millisecond scale. A qualitative difference can also be spotted with increasing power. At $100 \mu \mathrm{W}, g^{2}(\tau)$ remains constant along the normal at the millisecond scale-much like the stable emitter at all excitation powers. At higher excitation powers, starting from $500 \mu \mathrm{W}, g^{2}(\tau)$ shows additional bunching decay in the millisecond range [16]. This is direct evidence for a power-induced change in the emitters' photodynamics with an activation of the fourth state. Note that using a standard autocorrelation function at short delay times, this decay at millisecond time scales cannot be noticed, and will simply be manifested in a higher bunching [see Fig. 1(f)]. However, to describe this system precisely, an additional state in the system should be introduced. Using the $g^{2}(\tau)$ with double decay functions for excitation power in the range 500-2000 $\mu \mathrm{W}$, we determined characteristic decay times for the longer decay channel of 371, 222.4, 181.8, and $110.4 \mathrm{~ms}$ at 500, 1000, 1500, and $2000 \mu \mathrm{W}$, respectively. The decay time of the induced trap state decreases with increasing excitation power, showing rapid depopulation from the newly activated trap state at higher excitation powers. This is consistent with the observed reduction of blinking events with increasing power and similar intensity after blinking was induced [as in Fig. 2(a)].

Figure 3(c) shows the fluorescence photostability analysis corresponding to the blinking emitter displayed in Fig. 3(b): binning time is $50 \mathrm{~ms}$ and excitation powers are 100 , 1000 , and $2000 \mu \mathrm{W}$. As previously, to the right of each photostability time trace, the corresponding photon statistics is displayed, with 1000 photon binning. At $100 \mu \mathrm{W}$, the emitter showed stable emission with the intensity trace displaying no "dark" state interruptions as well as minimal photon statistics 
(a)

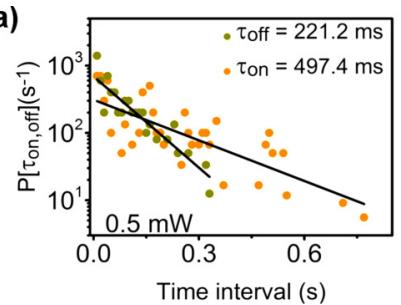

(c)

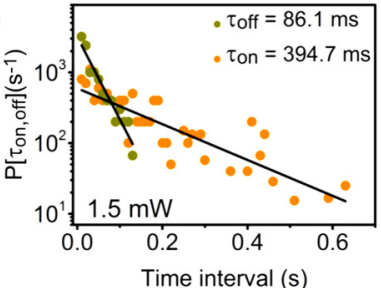

(b)

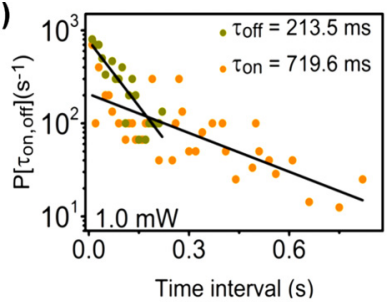

(d)

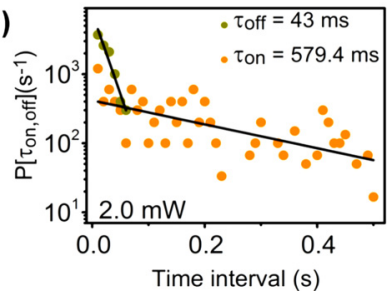

FIG. 4. Probability distribution of "on" (orange) and "off" (dark yellow) states of the blinking emitter at different excitation powers. (a)-(d) Semilogarithmic plots of the "on" and "off" time distributions of the fluorescence trajectories shown in Fig. 3(c). The probability distributions of both the "on" and "off" times at all excitation powers show exponential decay, as indicated by the linear trend on the semilogarithmic plots. The "off" probability distributions hold characteristic decay time $\left(\tau_{\text {off }}\right)$ that drop with increasing excitation power starting at $221.2,213.5,86.1$, and $43.1 \mathrm{~ms}$ for power excitation in the range 50-2000 $\mu \mathrm{W}$. Conversely the "on" time distribution did not show dependence on excitation power and gave a mean characteristic decay time $\left(\tau_{\text {on }}\right)$ of $(548 \pm 137) \mathrm{ms}$.

deviation from single state photon statistics. Upon further increase of the excitation power from 500 to $2000 \mu \mathrm{W}$, longtime-scale blinking set in with the fluorescence photostability showing clear "on" and "off" times. The alternating "on" and "off" events are observed on the photon statistics, with the occurrence of the "off" states decreasing with increasing excitation power.

To quantify the "on" and "off" times at different excitation powers, the probability density distribution $P\left[\tau_{\mathrm{on}}\right]$ and $P\left[\tau_{\mathrm{off}}\right]$ were plotted by setting a threshold intensity on the fluorescence trajectories of the blinking [Fig. 3(c)] [32]. In the fluorescence time trace, above and below the set threshold the emitter is considered to be "on" ( $\left.\tau_{\text {on }}\right)$ or "off” $\left(\tau_{\text {off }}\right)$, respectively.

$$
P\left(\tau_{\text {on }}, \tau_{\text {off }}\right) \approx \exp \left(\frac{-\tau}{\left(\tau_{\text {on }}, \tau_{\text {off }}\right)}\right) .
$$

This relation shows a linear distribution on log-linear plots as displayed in Fig. 4 for different excitation powers. Unlike

the widely reported power-law dependence of the probability density distribution on the $\tau_{\text {on }}$ and $\tau_{\text {off }}$ [23,32-34], single exponential decay of the form shown in Eq. (2) is observed for both "on" and "off" times in the emitters we analyzed [6,35]. From these plots, the characteristic decay times for $\tau_{\text {on }}$ and $\tau_{\text {off }}$ are determined for the excitation-dependent blinking, discussed above.

For excitation powers in the range $500-2000 \mu \mathrm{W}$, characteristic "off" time $\tau_{\text {off }}$, were measured to be $\sim 221.2,213.5$, 86.1 , and $43.1 \mathrm{~ms}$, respectively. The decline in the "off" times with increasing power is consistent with the fitting at long time scale of $g^{2}(\tau)$ and with the reduced blinking at high powers due to faster depopulation from the activated trap state.

Conversely, the probability distribution of "on" times did not show significant dependence on the excitation power, yielding a mean "on" time $\left(\tau_{\mathrm{on}}\right)$ of $(548 \pm 137) \mathrm{ms}$. The weak dependence was also observed on the florescence statistics, where the "on" event occurrence remained the same while the "off" event decreased. This suggests that the depopulation of the new trap state possibly leads to a population of another shelving state. This is indicated by the rate coefficient $\kappa_{43}$ in Fig. S4 [17]. However, because the original shelving state and the induced trap state are weakly coupled in time, as demonstrated already via the second-order autocorrelation function analysis of the transition kinetics, the effective increase in "on" times is not significant.

In conclusion, we presented a comprehensive investigation power-dependent blinking of SPEs in GaN. We demonstrated that the excitation power permanently activates trapping states which act as additional shelving states associated with blinking. This is in contrast to known emitters in solids that, upon higher excitation, populate the same shelving/metastable state, or quantum dots and single molecules that tend to bleach shortly after blinking. Our work also has practical implications. For example, the fact that the "on" time state remains relatively unchanged, means that the emitters in $\mathrm{GaN}$ can be used in practical quantum photonic applications (e.g., two-photon interference) where high photon flux is important [36,37]. Overall, our work helps to shed more light onto a rather complicated phenomenon-blinking in solid-state SPEs-and emphasizes that standard three-level models may not always be ideal to describe the photodynamics of such systems.

Financial support from the Australian Research Council (via Grant No. DE130100592) and the Asian Office of Aerospace Research and Development (Grant No. FA2386-15$1-4044)$ is gratefully acknowledged. The authors thank Milos Toth and Bernd Sontheimer for useful discussions.
[1] A. L. Efros and D. J. Nesbitt, Origin and control of blinking in quantum dots, Nat. Nanotechnol. 11, 661 (2016).

[2] P. Frantsuzov, M. Kuno, B. Janko, and R. A. Marcus, Universal emission intermittency in quantum dots, nanorods and nanowires, Nat. Phys. 4, 519 (2008).

[3] F. D. Stefani, J. P. Hoogenboom, and E. Barkai, Beyond quantum jumps: Blinking nanoscale light emitters, Phys. Today 62(2), 34 (2009).

[4] C. Bradac, T. Gaebel, N. Naidoo, M. J. Sellars, J. Twamley, L. J. Brown, A. S. Barnard, T. Plakhotnik, A. V. Zvyagin, and
J. R. Rabeau, Observation and control of blinking nitrogenvacancy centres in discrete nanodiamonds, Nat. Nanotechnol. 5, 345 (2010).

[5] E. Neu, M. Agio, and C. Becher, Photophysics of single silicon vacancy centers in diamond: Implications for single photon emission, Opt. Express 20, 19956 (2012).

[6] S. Castelletto, B. Johnson, V. Ivády, N. Stavrias, T. Umeda, A. Gali, and T. Ohshima, A silicon carbide room-temperature single-photon source, Nat. Mater. 13, 151 (2014). 
[7] E. Barkai, Y. Jung, and R. Silbey, Theory of single-molecule spectroscopy: Beyond the ensemble average, Annu. Rev. Phys. Chem. 55, 457 (2004).

[8] T. Gensch, M. Böhmer, and P. F. Aramendía, Single molecule blinking and photobleaching separated by wide-field fluorescence microscopy, J. Phys. Chem. A 109, 6652 (2005).

[9] V. Rombach-Riegraf, P. Oswald, R. Bienert, J. Petersen, M. Domingo, J. Pardo, P. Gräber, and E. Galvez, Blinking effect and the use of quantum dots in single molecule spectroscopy, Biochem. Biophys. Res. Commun. 430, 260 (2013).

[10] B. Mahler, P. Spinicelli, S. Buil, X. Quelin, J.-P. Hermier, and B. Dubertret, Towards non-blinking colloidal quantum dots, Nat. Mater. 7, 659 (2008).

[11] K. T. Shimizu, R. G. Neuhauser, C. A. Leatherdale, S. A. Empedocles, W. Woo, and M. G. Bawendi, Blinking statistics in single semiconductor nanocrystal quantum dots, Phys. Rev. B 63, 205316 (2001).

[12] J. N. Clifford, T. D. M. Bell, P. Tinnefeld, M. Heilemann, S. M. Melnikov, J.-I. Hotta, M. Sliwa, P. Dedecker, M. Sauer, and J. Hofkens, Fluorescence of single molecules in polymer films: Sensitivity of blinking to local environment, J. Phys. Chem. B 111, 6987 (2007).

[13] G. Messin, J.-P. Hermier, E. Giacobino, P. Desbiolles, and M. Dahan, Bunching and antibunching in the fluorescence of semiconductor nanocrystals, Opt. Lett. 26, 1891 (2001).

[14] W.-T. Yip, D. Hu, J. Yu, D. A. Vanden Bout, and P. F. Barbara, Classifying the photophysical dynamics of single- and multiplechromophoric molecules by single molecule spectroscopy, J. Phys. Chem. A 102, 7564 (1998).

[15] A. M. Berhane, K.-Y. Jeong, Z. Bodrog, S. Fiedler, T. Schröder, N. V. Triviño, T. Palacios, A. Gali, M. Toth, D. Englund, and I. Aharonovich, Bright room-temperature single-photon emission from defects in gallium nitride, Adv. Mater. 29, 1605092 (2017).

[16] O. Neitzke, A. Morfa, J. Wolters, A. W. Schell, G. N. Kewes, and O. Benson, Investigation of line width narrowing and spectral jumps of single stable defect centers in $\mathrm{ZnO}$ at cryogenic temperature, Nano Lett. 15, 3024 (2015).

[17] See Supplemental Material at http://link.aps.org/supplemental/ 10.1103/PhysRevB.96.041203 for all the experimental details, supporting data, and analysis of transition kinetics.

[18] B. Szûcs, A. Gali, Z. Hajnal, P. Deák, and C. G. Van de Walle, Physics and chemistry of hydrogen in the vacancies of semiconductors, Phys. Rev. B 68, 085202 (2003).

[19] C. G. Van de Walle, Interactions of hydrogen with native defects in GaN, Phys. Rev. B 56, 10020(R) (1997).

[20] K. Han, D. Wildanger, E. Rittweger, J. Meijer, S. Pezzagna, S. Hell, and C. Eggeling, Dark state photophysics of nitrogenvacancy centres in diamond, New J. Phys. 14, 123002 (2012).

[21] E. Rittweger, D. Wildanger, and S. Hell, Far-field fluorescence nanoscopy of diamond color centers by ground state depletion, Europhys. Lett. 86, 14001 (2009).

[22] T. Plakhotnik and R. Chapman, Nitrogen-vacancy centers in nano-diamond reversibly decrease the luminescence quantum yield under strong pulsed-laser irradiation, New J. Phys. 13, 045001 (2011).
[23] P. A. Frantsuzov and R. A. Marcus, Explanation of quantum dot blinking without the long-lived trap hypothesis, Phys. Rev. B 72, 155321 (2005)

[24] N. I. Hammer, K. T. Early, K. Sill, M. Y. Odoi, T. Emrick, and M. D. Barnes, Coverage-mediated suppression of blinking in solid state quantum dot conjugated organic composite nanostructures, J. Phys. Chem. B 110, 14167 (2006).

[25] S.-J. Park, S. Link, W. L. Miller, A. Gesquiere, and P. F. Barbara, Effect of electric field on the photoluminescence intensity of single CdSe nanocrystals, Chem. Phys. 341, 169 (2007).

[26] E. Neu, D. Steinmetz, J. Riedrich-Möller, S. Gsell, M. Fischer, M. Schreck, and C. Becher, Single photon emission from siliconvacancy colour centres in chemical vapour deposition nanodiamonds on iridium, New J. Phys. 13, 025012 (2011).

[27] C. Kurtsiefer, S. Mayer, P. Zarda, and H. Weinfurter, Stable Solid-State Source of Single Photons, Phys. Rev. Lett. 85, 290 (2000).

[28] S. Kitson, P. Jonsson, J. Rarity, and P. Tapster, Intensity fluctuation spectroscopy of small numbers of dye molecules in a microcavity, Phys. Rev. A 58, 620 (1998).

[29] E. Wu, V. Jacques, H. Zeng, P. Grangier, F. Treussart, and J.-F. Roch, Narrow-band single-photon emission in the near infrared for quantum key distribution, Opt. Express 14, 1296 (2006).

[30] I. Aharonovich, S. Castelletto, D. A. Simpson, A. D. Greentree, and S. Prawer, Photophysics of chromium-related diamond single-photon emitters, Phys. Rev. A 81, 043813 (2010).

[31] T. T. Tran, C. Elbadawi, D. Totonjian, C. J. Lobo, G. Grosso, H. Moon, D. R. Englund, M. J. Ford, I. Aharonovich, and M. Toth, Robust multicolor single photon emission from point defects in hexagonal boron nitride, ACS Nano 10, 7331 (2016).

[32] M. Kuno, D. P. Fromm, H. F. Hamann, A. Gallagher, and D. J. Nesbitt, Nonexponential "blinking" kinetics of single CdSe quantum dots: A universal power law behavior, J. Chem. Phys 112, 3117 (2000).

[33] J. Tang and R. A. Marcus, Diffusion-Controlled Electron Transfer Processes and Power-Law Statistics of Fluorescence Intermittency of Nanoparticles, Phys. Rev. Lett. 95, 107401 (2005).

[34] R. Verberk, A. M. van Oijen, and M. Orrit, Simple model for the power-law blinking of single semiconductor nanocrystals, Phys. Rev. B 66, 233202 (2002).

[35] A. L. Efros and M. Rosen, Random Telegraph Signal in the Photoluminescence Intensity of a Single Quantum Dot, Phys. Rev. Lett. 78, 1110 (1997).

[36] A. Sipahigil, K. D. Jahnke, L. J. Rogers, T. Teraji, J. Isoya, A. S. Zibrov, F. Jelezko, and M. D. Lukin, Indistinguishable Photons from Separated Silicon-Vacancy Centers in Diamond, Phys. Rev. Lett. 113, 113602 (2014).

[37] A. Batalov, C. Zierl, T. Gaebel, P. Neumann, I.-Y. Chan, G. Balasubramanian, P. Hemmer, F. Jelezko, and J. Wrachtrup, Temporal Coherence of Photons Emitted by Single NitrogenVacancy Defect Centers in Diamond Using Optical RabiOscillations, Phys. Rev. Lett. 100, 077401 (2008). 